Research Article

\title{
Wind Tunnel Tests and Numerical Simulations of Wind-Induced Snow Drift in an Open Stadium and Gymnasium
}

\author{
Xintong Jiang $\mathbb{D}^{\mathbb{D}}$, Zhixiang Yin $\left(\mathbb{D}\right.$, and Hanbo Cui $\mathbb{D}^{\circ}$ \\ School of Mechanics and Engineering, Liaoning Technical University, Fuxin, Liaoning 123000, China \\ Correspondence should be addressed to Hanbo Cui; lgdchb@sina.com
}

Received 8 March 2020; Revised 28 October 2020; Accepted 29 October 2020; Published 29 November 2020

Academic Editor: Hui Yao

Copyright (C) 2020 Xintong Jiang et al. This is an open access article distributed under the Creative Commons Attribution License, which permits unrestricted use, distribution, and reproduction in any medium, provided the original work is properly cited.

\begin{abstract}
A long-span sports centre generally comprises multiple stadiums and gymnasiums, for which mutual interference effects of windinduced snow motion are not explicitly included in the specifications of various countries. This problem is addressed herein by performing wind tunnel tests and numerical simulations to investigate the snow distribution and mutual interference effect on the roofs of long-span stadiums and gymnasiums. The wind tunnel tests were used to analyse the influences of the opening direction $\left(0^{\circ}, 90^{\circ}, 180^{\circ}\right.$, and $\left.270^{\circ}\right)$ and spacing $(0.3 \mathrm{~L}, 0.5 \mathrm{~L}, 1 \mathrm{~L}, 1.5 \mathrm{~L}, 2 \mathrm{~L}$, and $2.5 \mathrm{~L}$, where $\mathrm{L}$ is the gymnasium span) of the stadium and gymnasium. The wind tunnel tests and numerical simulations were used to analyse the influence of the wind direction angle (from $0^{\circ}$ to $315^{\circ}$, there are a total of eight groups in $45^{\circ}$ intervals). The following results were obtained. The stadium opening had a significant effect on the snow distribution on the surface of the two structures. An even snow distribution was obtained when the stadium opened directly facing the gymnasium, which corresponded to the safest condition for the structures' surfaces. As the spacing between the buildings increased, the interference effect between the two structures was reduced. The interference was negligible for a spacing of $2 \mathrm{~L}$. The stadium had the most significant amplification interference effect on the gymnasium for a wind direction angle of $45^{\circ}$, which was extremely unfavourable to the safety of the structure. The most favourable wind direction angle was $270^{\circ}$, where there were both amplification interference and blockage interference.
\end{abstract}

\section{Introduction}

In the recent years, a continuous increase in the scale of sporting events and improved living standards has led to a gradual increase in the number of sports centres that consist of long-span gymnasiums and long-span stadiums. This combination offers a wider range of functionalities and more open space and can better fulfill people's needs. There are openings on the surface or the main body of many stadiums, such as the Munich Olympic Stadium, the London Olympic Stadium, and the Shanghai Stadium. These buildings have sensitive structures, and the pressure gradient at the building opening drives complex wind flows that affect the trajectory of snow, resulting in uneven snow distribution. The blockage effect from two buildings produces complex wind-induced snow motion between the buildings. However, there is currently no explicit standard for mutual interference effects in the codes of various countries. To address this problem, we used the sports centre in Huludao, China, as a case study to investigate the snow distribution on structure surfaces. Wind tunnel tests and numerical simulations were used to determine the mutual interference effects of the two buildings and to predict the snow distribution on the surfaces of buildings with openings.

Wind-induced snow drift has been investigated using theoretical studies (Schmidt [1]; Kind [2]; and Cocca and O'Rourke [3]), field measurements (O'Rourke et al. [4]; Oikawa et al. [5]; Thiis [6]; and Tomabechi et al. [7]), wind tunnel tests, and numerical simulations. Wind tunnel tests are usually scaled tests, in which the selection of appropriate similarity parameters is critical. In practical tests, only the main similarity parameters can be matched. Many researchers (Kind [8]; Tabler [9]; Iversen [10]; and Anno [11]) have studied geometric similarity, motion similarity, dynamic similarity, and time scale similarity. The Froude and Reynolds numbers are also two important parameters, but 
they can produce contradictory requirements. Some researchers (Anno [11]; Kim et al. [12]; and Kwok et al. [13]) believe that the Froude number requirements can be relaxed, whereas others (Lever and Haehnel [14]) have concluded that the Froude number can be neglected when the particle saltation height is much smaller than the model size. Some researchers have studied the factors that affect wind-induced snow drift. Peterka and Esterday [15] conducted a wind tunnel test on the snow distribution law of the model surface area under different wind directions and ultimately determined the roof snow load. Zhou et al. $[16,17]$ studied the snow distribution law of the surface area of flat roofs with different span-to-height ratios under different wind speeds and blowing times and considered the relationship between the particle mass transport rate and the transport volume. $\mathrm{Yu}$ et al. [18] conducted wind tunnel tests on a flat threedimensional (3D) roof and considered the effects of the model size, wind speed, and blowing time. Qiang et al. [19] conducted snow tests on flat roofs in a low-temperature wind tunnel environment and simulated the real snowfall process, including during snowfall and after snowfall, and put forward a formula to calculate the snow transport rate.

Unlike wind tunnel tests, full-scale models can be considered in numerical simulations, which can, in turn, be used to solve the similarity problems. Numerical simulations are inexpensive and rapid, and they do not require special equipment. Therefore, numerical simulations have become one of the most effective methods for studying wind-induced snow drift, although the simulation results need to be verified by field measurements or wind tunnel tests. Many researchers have made improvements in the accuracy of numerical simulations. Sato et al. [20] simulated 3D windinduced snow drift by considering snow saltation and suspension. The finite volume method was used to solve the problem and produced results that were consistent with the measurements. Beyers and Waechter [21] used an improved turbulent wall function to account for the additional momentum from snow particle saltation motion. A simulation of the snow drift around a cubic model produced satisfactory results. Zhao et al. [22] studied bidirectional coupled windinduced snow flow using a numerical simulation method based on CFD-DEM modelling. A two-dimensional (2D) numerical simulation was performed for the snow distribution and wind-snow coupling interference effect on a typical stepped roof. Liu et al. [23] introduced two novel parameters, the snow residual rate and the erosion rate, in wind tunnel tests on flat 3D roofs. Numerical simulations were used to correlate the vorticity magnitude with the friction velocity around a structure. Wang et al. [24] combined wind tunnel tests and numerical simulations to develop a probability-based half-steady Eulerian-Lagrangian method for studying the snow distribution on roofs. Marsh et al. [25] proposed a 3D snow blowing model that uses a variable resolution unstructured grid for spatial discretization. With a fixed-resolution grid size, computational elements are reduced by $62 \%$, and the total run time is reduced by $44 \%$.

In most of the current literature, only single closed structures have been studied. In the present study, an actual project is used as a case study to investigate the snow distribution on the surface of adjacent long-span open structures. There are many factors that affect the snow distribution on the surface, as shown in Figure 1. He et al. [26] pointed out that the wind effects around different buildings could overlap, interact, neutralize, and offset. This leads to changes in the trajectory of the snow particles around the building, which affects the final snow distribution. The direction of the openings and the spacing between structures are both factors that affect the arrangement of structures, and thus, the influence of these two factors on the distribution of snow cover can be studied. Additionally, the influence of the wind direction angle on the mutual interference effect of the two structures is studied. The obtained results are realistic and can be used to predict the snow distribution for buildings with openings.

\section{Wind Tunnel Tests}

2.1. Description of the Test. A small, low-speed wind tunnel was designed and used to perform tests on wind-induced snow drifts [27]. The wind tunnel had a total length of seven metres, and the maximum airflow was $18,700\left(\mathrm{~m}^{3} / \mathrm{h}\right)$. The wind turbine was connected to a CDI-EM60G2R2T4B frequency converter to continuously adjust the wind speed. Measurements showed that the wind speed was linearly proportional to the frequency, and the steady wind speed in the tests was 1.5 to $10 \mathrm{~m} / \mathrm{s}$. The test section had dimensions of $0.5 \mathrm{~m} \times 0.5 \mathrm{~m} \times 1.5 \mathrm{~m}$. A simple box was placed at the outlet of the diffuser section to determine the mass transport rate. In the wind tunnel test section, the airflow stability did not exceed $1.5 \%$, and the dynamic pressure field coefficient was below two percent. Taking a height of $10 \mathrm{~cm}$ as the reference point, the measured wind profile and turbulence intensity profile at the nominal velocity of $5 \mathrm{~m} / \mathrm{s}$ are shown in Figure 2. As seen from Figure 2(a), the wind velocity of the flow field $u$ was strongly influenced by the wall height $z$. The wind velocity near the wall was relatively small, and it increased with $z$. Within a height of $0.1 \mathrm{~m}$, the increase of $u$ with $z$ was relatively large. When the wall height $z$ was greater than $0.1 \mathrm{~m}, u$ increased with $z$ steadily. From Figure 2(b), it can be seen that the turbulence intensity $I$ decreased with $z$. The position that had the maximum value of $I$ (approximately 5.5-6.0\%) was at the bottom of the flow field. A turbulence curve was proposed from Figure 2 to show the relationship between the wind velocity profile and the turbulence intensity profile. The fitting relationship was $I=5.886-0.002 u^{4.315}$, and $R^{2}=0.990$, as shown in Figure 3. It can be seen from Figure 3 that the turbulence intensity I at a height of $0.1 \mathrm{~m}$ decreased with an increase in the wind velocity $u$. This was because the higher the wind velocity was, the more particles moved. The leaping particles continuously absorb energy from the airflow to reduce the pulsation velocity and turbulence intensity. The logarithm law was used to fit the wind velocity curve, and the obtained roughness height $z_{o}$ was $1.4 \times 10^{-4} \mathrm{~m}$. Roughness elements were laid at the front of the test section to fully develop the wind. The prepavement method was used to simulate building the surface 


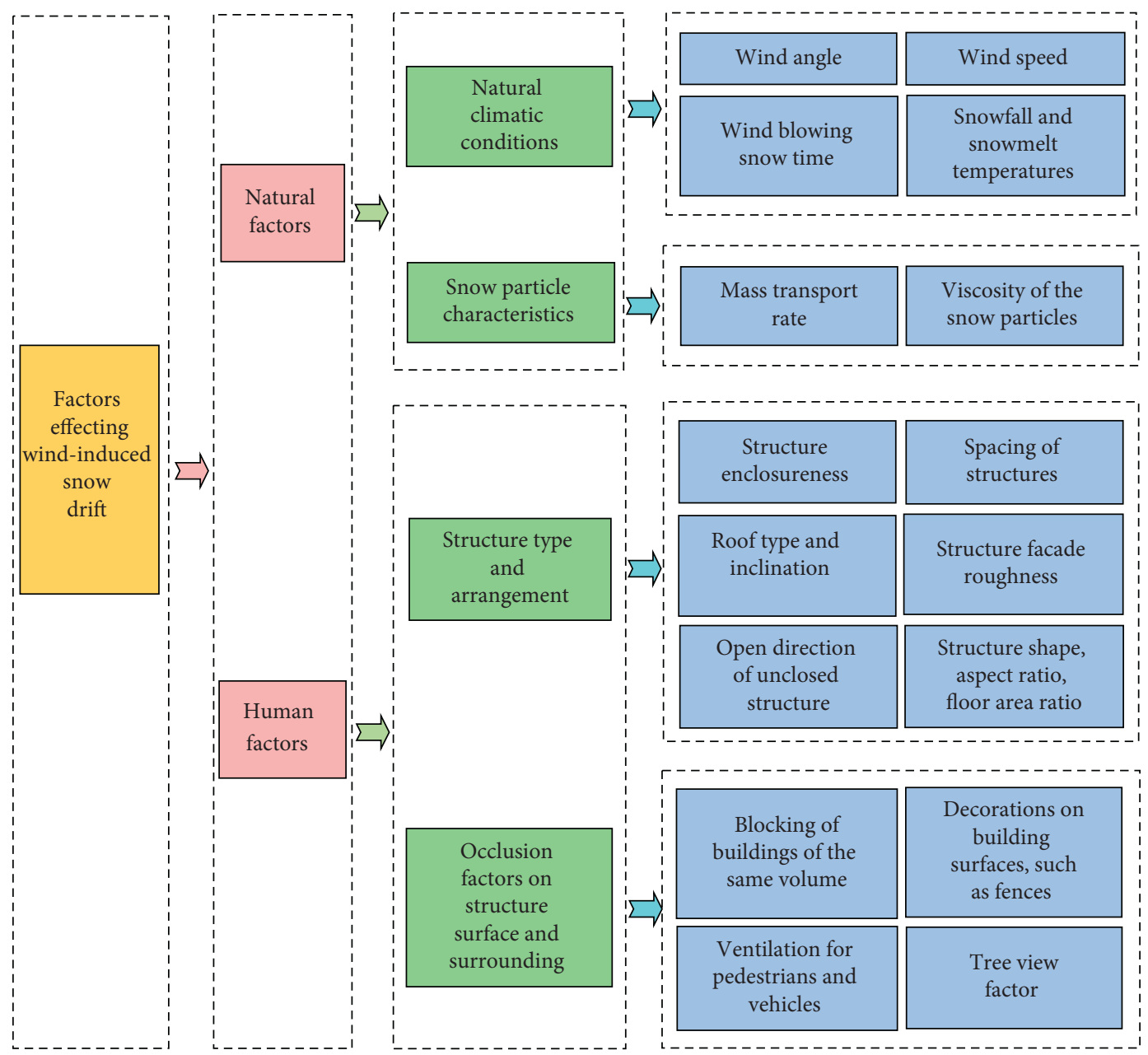

FIGURE 1: Factors effecting wind-induced snow drift $([18,19,26])$.

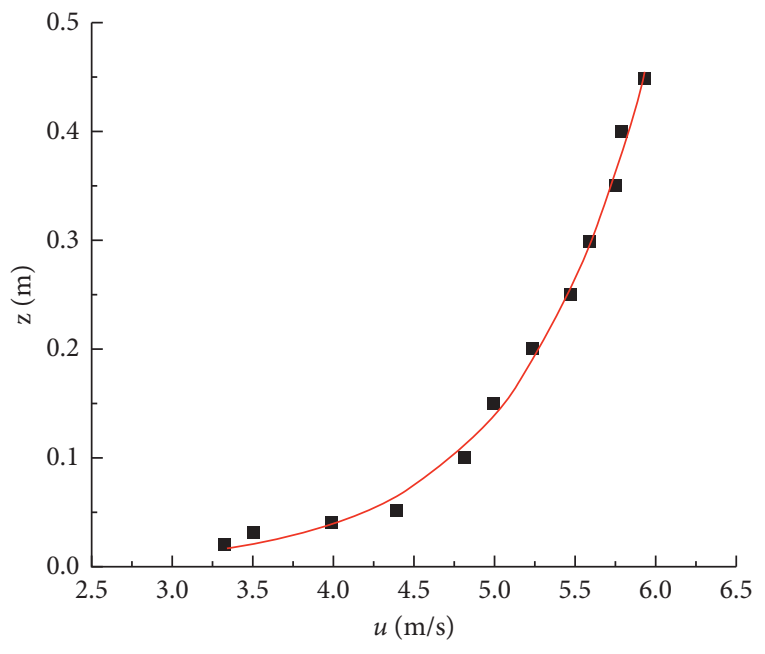

- Test results

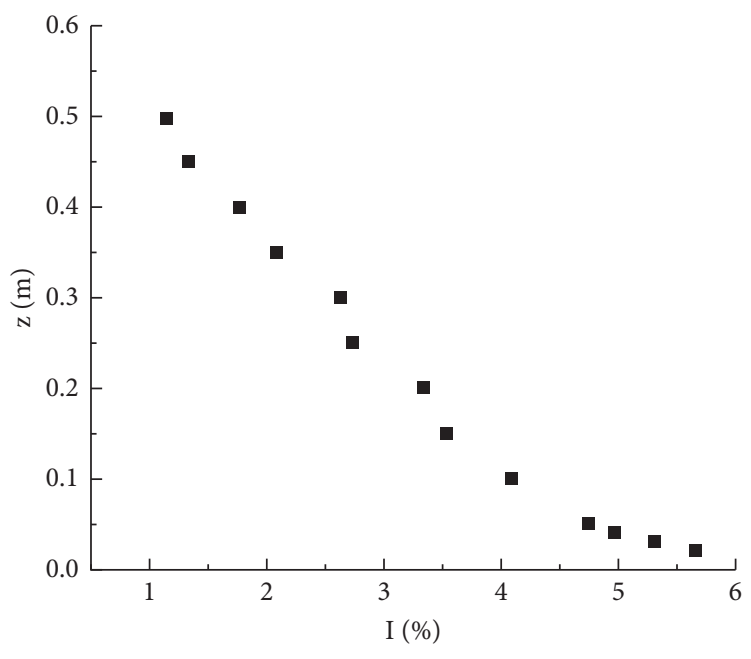

(a)

(b)

Figure 2: Profiles of the mean wind and turbulence intensity. (a) Wind velocity profile. (b) Turbulence intensity profile. 


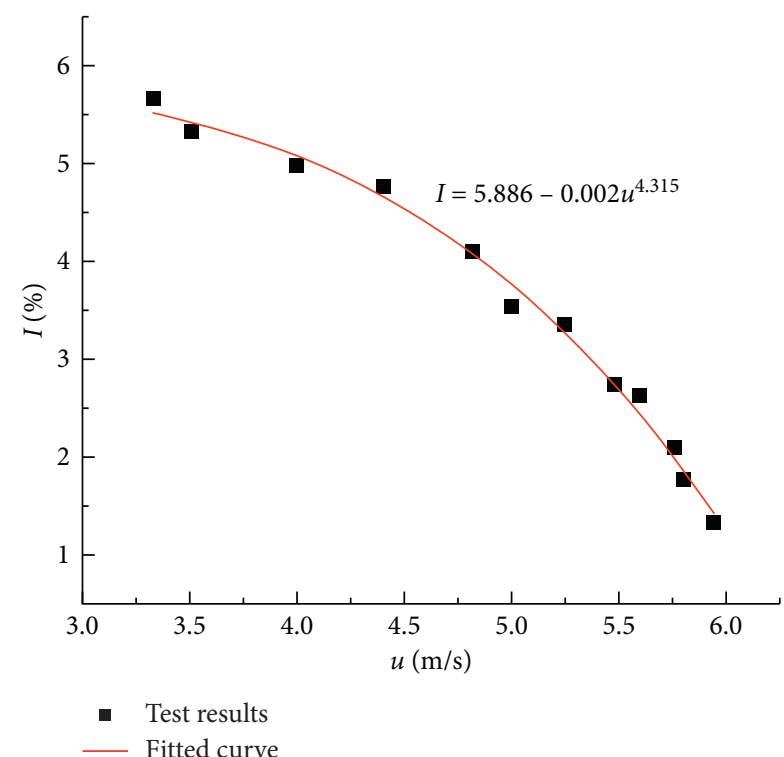

FIGURE 3: Fitting curve of the turbulence intensity and wind velocity.

snow. An infrared rangefinder was installed on a fixed slide to measure the snow thickness to an accuracy of one millimetre. The snow depth coefficient $C_{s}$ was obtained via dimensionless transformation of the snow depth after the test. The variation in $C_{s}$ reflected the snow distribution on the surface.

2.2. Similarity Parameters and Particle Selection. Geometric similarity, motion similarity, dynamic similarity, Reynolds number similarity, and time scale similarity were used to ensure the accuracy of the test results. Similarity of the angle of repose was used to determine the similarity of the snow particles. Many researchers have obtained satisfactory results by using quartz sand in wind-snow tests (Kind [28]; Li [29]; Wang et al. [30]; and Zhou et al. [17, 31]). This medium was also used in this study. The mean diameter of the measured quartz sand was $0.14 \mathrm{~mm}$, the density was $2.56\left(\mathrm{~g} / \mathrm{cm}^{3}\right)$, and the angle of repose was $30.8^{\circ}$. Table 1 shows that quartz sand and snow particles essentially have similar physical properties. As $w_{f}$ was greater than the prototype value, $\left(w_{f} / u_{*}\right)$ and $\left(w_{f} / u_{* t}\right)$ were both two to three times larger than the prototype values. The Froude number of the model was two orders of magnitude higher than that of the prototype and was inaccurately simulated. Several researchers have shown that the Reynolds numbers and incompatible Froude numbers are more suitable for wind-induced snow drift wind tunnel tests. The meteorological data of the 2007 blizzard in Liaoning was used as a case study (Li et al. [32]): the duration of this blizzard was $48 \mathrm{~h}$, and the force 8-9 north-easterly wind had a wind velocity of between 17 and $24 \mathrm{~m} / \mathrm{s}$, with an average wind velocity of $20.5 \mathrm{~m} / \mathrm{s}$. We adopt the test criterion proposed by Iversen [10], which is based on the similarity of the mass transport rate of the saltation layer, as given by equations (1) and (2). The test wind velocity was $5.2 \mathrm{~m} / \mathrm{s}$, and the test duration was thirteen minutes.
TABLE 1: Main parameters of the particles.

\begin{tabular}{lcc}
\hline Parameters & Model values & Prototype values \\
\hline$\left(u_{* t}\right)(\mathrm{m} / \mathrm{s})$ & 0.14 & $0.118 \sim 0.28$ \\
$\left(w_{f}\right)(\mathrm{m} / \mathrm{s})$ & 2 & $0.31 \sim 0.75$ \\
$u_{* t}^{3} /(2 g v)$ & 9.66 & $7 \sim 70$ \\
$\left(u_{*} / u_{* t}\right)$ & $1.31 \sim 1.69$ & $0.66 \sim 7.93$ \\
$\left(w_{f} / u_{*}\right)$ & $8.44 \sim 10.91$ & $0.3 \sim 3.9$ \\
$\left(u_{*}^{2} / L g\right)$ & $0.013 \sim 0.02$ & $0.00043 \sim 0.00072$ \\
$\left(w_{f} / u_{* t}\right)$ & 14.28 & $2.16 \sim 5$ \\
\hline
\end{tabular}

In Table $1, u_{* t}$ is the threshold friction velocity $(\mathrm{m} / \mathrm{s}), w_{f}$ is the particle settling velocity $(\mathrm{m} / \mathrm{s}), v$ is the kinematic viscosity of the fluid $(\mathrm{m} / \mathrm{s})$, and $u_{*}$ is the friction velocity $(\mathrm{m} / \mathrm{s})$.

$$
\begin{aligned}
{\left[\frac{\rho}{\rho_{p}} \frac{U^{2}}{g L}\left(1-\frac{U_{0}}{U}\right)\right]_{m} } & =\left[\frac{\rho}{\rho_{p}} \frac{U^{2}}{g L}\left(1-\frac{U_{0}}{U}\right)\right]_{p} \\
\left(\frac{U T}{L}\right)_{m} & =\left(\frac{U T}{L}\right)_{p},
\end{aligned}
$$

where $\rho$ is the air density $\left(\mathrm{kg} / \mathrm{m}^{3}\right), \rho_{p}$ is the snow density $(\mathrm{kg} /$ $\left.\mathrm{m}^{3}\right), U$ is the reference point wind velocity $(\mathrm{m} / \mathrm{s}), U_{0}$ is the initial particle speed $(\mathrm{m} / \mathrm{s}), L$ is the geometric scale $(\mathrm{m}), g$ is the acceleration due to gravity $\left(\mathrm{m} / \mathrm{s}^{2}\right)$, and $T$ is the time for the snowfall (s).

2.3. Test Model. The prototype building consists of a longspan stadium with an open end and an oval long-span gymnasium. The stadium has a $246 \mathrm{~m}$ long axis and a $224 \mathrm{~m}$ short axis, and the highest roof elevation is $49 \mathrm{~m}$. The gymnasium has a $135 \mathrm{~m}$ long axis and a $116 \mathrm{~m}$ short axis, and the highest roof elevation is $24 \mathrm{~m}$. The buildings are shown in Figure 4 . The test model is a 1/700 scale and is made of welded 304 stainless steel with a thickness of two millimetres. Figures 5 and 6 show two groups of 91 and 69 measurement points on the stadium surface and two groups of 92 and 68 measuring points on the gymnasium surface, respectively. Similar variations in $C_{s}$ are found using both sets of measurement points (there were a few numerical differences between the $C_{s}$ plots). Therefore, we select the group of 69 measurement points on the stadium surface and the group of 68 measuring points on the gymnasium surface for analysis. Figure 7 shows the test results for a stadium opening direction of $90^{\circ}$, a $0.5 \mathrm{~L}$ spacing between the two buildings, and a wind direction angle of $90^{\circ}$.

\section{Numerical Simulations}

3.1. Governing Equation of the Air Phase and Snow Phase. Using the Reynolds average method, wind can be approximated as an incompressible viscous fluid, and the continuous equation and the momentum conservation equation are given by the following equations, respectively: 


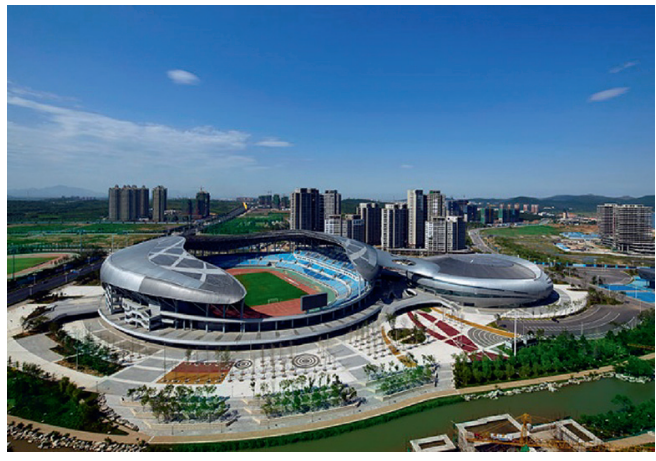

Figure 4: Huludao sports centre.

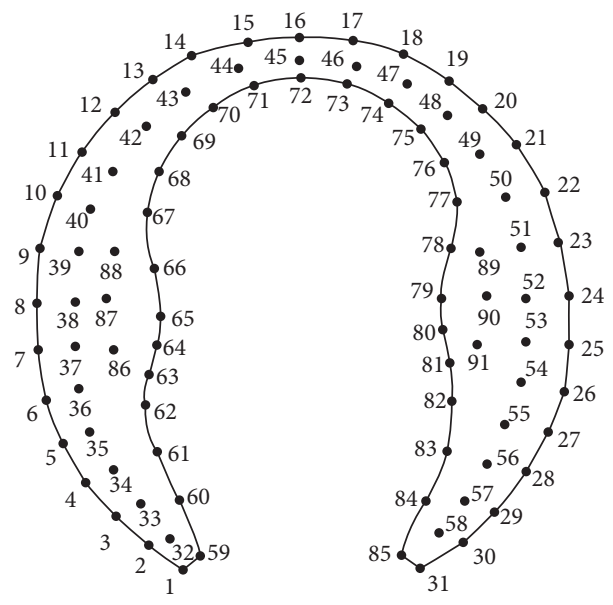

(a)

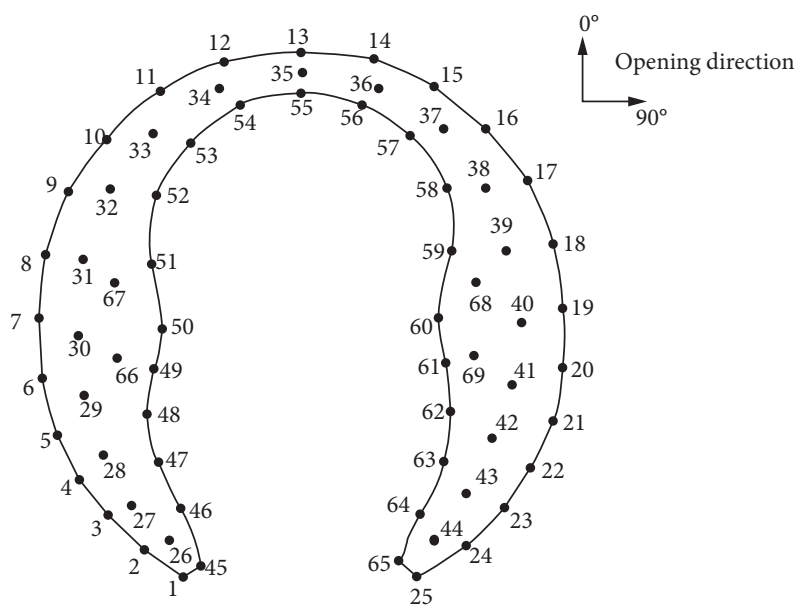

(b)

FIGURE 5: Layout of the stadium monitoring points. (a) 91 measurement points. (b) 69 measurement points.

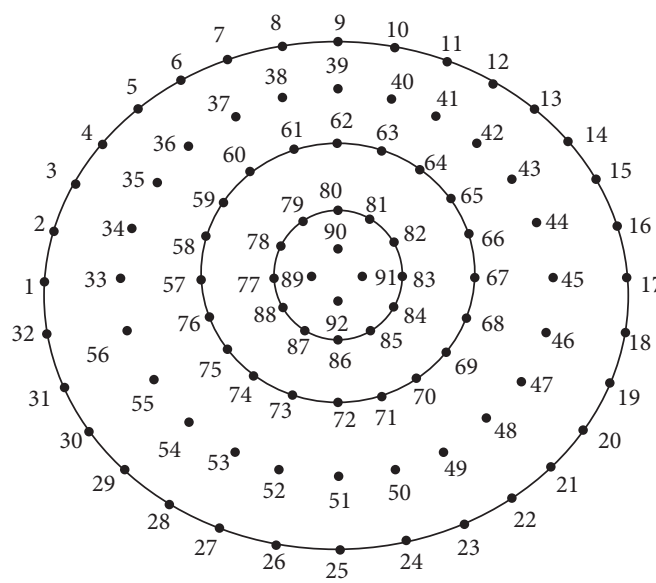

(a)

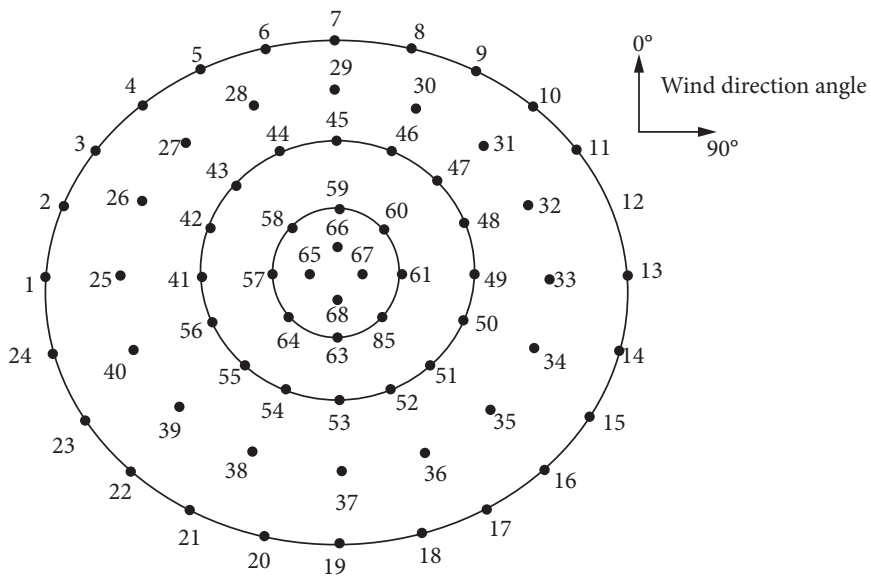

(b)

FIGURE 6: Layout of the gymnasium measuring points. (a) 92 measuring points. (b) 68 measuring points. 


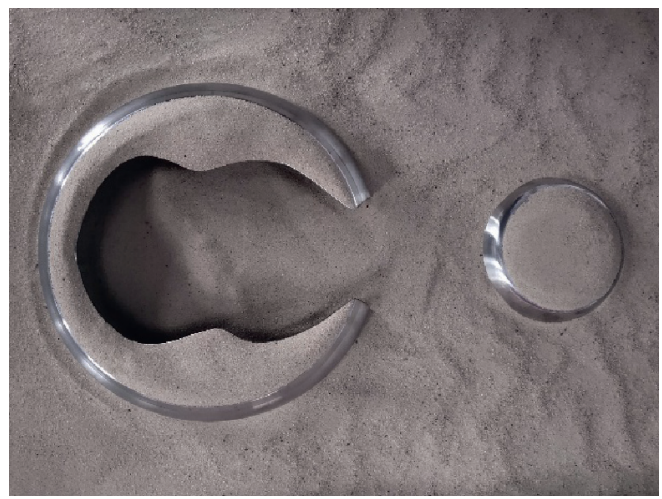

FIgURe 7: Photographs of the test results.

$$
\begin{aligned}
& \frac{\partial u}{\partial x}+\frac{\partial v}{\partial y}+\frac{\partial w}{\partial z}=0 \\
& \frac{D u_{i}}{D t}=f_{i}-\frac{1}{\rho} \frac{\partial p}{\partial x_{i}}+\frac{\mu}{\rho} \frac{\partial}{\partial x_{j}}\left(\frac{\partial u_{i}}{\partial x_{j}}\right)
\end{aligned}
$$

where $u, v$, and $w$ are the velocity components in the directions of $x, y$, and $z$, respectively, $u_{i}$ is the wind velocity component $(\mathrm{m} / \mathrm{s}), t$ is the time component $(\mathrm{s}), f_{i}$ is the component of the force acting on a unit mass of the fluid $(\mathrm{N})$, $x_{i}$ and $x_{j}$ are coordinates, $p$ is the fluid pressure $(\mathrm{MPa})$, and $\mu$ is the air viscosity coefficient $(\mathrm{Pa} \cdot \mathrm{s})$.

The governing equation of the snow phase (Zhou et al. [16]; Uematsu et al. [33]; and Tominaga et al. [34]) is given by the following equation:

$$
\begin{gathered}
\frac{\partial\left(\rho_{p} f\right)}{\partial t}+\frac{\partial\left(\rho_{p} f u_{j}\right)}{\partial x_{j}}=\frac{\partial}{\partial x_{j}}\left(\mu_{t} \frac{\partial \rho_{p} f}{\partial x_{j}}\right) \\
+\frac{\partial}{\partial x_{j}}\left(-\rho_{p} f u_{R, j}\right),
\end{gathered}
$$

where $f$ is the mass concentration of snow, $u_{j}$ is the wind velocity vector $(\mathrm{m} / \mathrm{s}), \mu_{t}$ is the turbulent kinematic viscosity, which represents the degree of influence of air on the snow faces, and the speed of snow relative to the air phase $u_{\mathrm{R}, j}$ is $0.3[35]$.

3.2. Snow Erosion and Deposition. The friction velocity $u_{*}$ is the most important parameter for studying wind-induced snow drift:

$$
u_{*}=\frac{u(z) K}{\ln \left(z / z_{o}\right)},
$$

where $u(z)(\mathrm{m} / \mathrm{s})$ is the reference wind velocity at a height of $z(\mathrm{~m})$, and the von Karman constant $K$ is 0.40 . The value of $u_{*}$ relative to the threshold friction velocity $u_{* t}$ determines if the snow is eroded or deposited. For $u_{*} \geq u_{* t}$, the snow particles enter the watershed, and snow on the surface is eroded, whereas for $u_{*}<u_{* t}$, drifting snow particles fall and are deposited onto the surface. The snow erosion per unit area per unit time $q_{\text {ero }}$ and the amount of deposition $q_{\text {dep }}$ are given by the following equations, respectively:

$$
\begin{gathered}
q_{\text {ero }}=A_{\text {ero }}\left(u_{* t}^{2}-u_{*}^{2}\right)\left(u_{*} \geq u_{* t}\right), \\
q_{\text {dep }}=C w_{f} \frac{u_{* t}^{2}-u_{*}^{2}}{u_{* t}^{2}}\left(u_{*}<u_{* t}\right),
\end{gathered}
$$

where $A_{\text {ero }}$ is a constant coefficient, which is taken as $7.0 e-4$, and $C$ is the mass of snow within a unit volume, $C=f \rho_{p}$.

3.3. Calculation Model and Meshing. FLUENT software was used to perform the numerical simulations on the stadium and gymnasium. Unidirectional coupling was assumed between the air and snow particles; that is, air can drive the motion of snow particles, but the drift of snow particles does not affect the air. The Euler-Euler method was used. A mixture multiphase flow model was adopted for its relative stability at a low computational cost. A fullscale simulation was performed. The watershed had dimensions of $3000 \mathrm{~m} \times 1500 \mathrm{~m} \times 500 \mathrm{~m}$. The model was placed in the first third of the watershed. Hybrid meshing was adopted, that is, an unstructured mesh was used to discretise the building, and a structured mesh was used to discretise the exterior space, resulting in a total number of approximately 1.7 million meshes.

3.4. Boundary Conditions and the Solution Method. The velocity inlet boundary condition for the air phase on the inflow surface is

$$
u(z)=u_{b}\left(\frac{z}{z_{b}}\right)^{\alpha},
$$

where $u_{b}(\mathrm{~m} / \mathrm{s})$ is the wind velocity at a standard reference height of $z_{b}(\mathrm{~m}), z_{b}$ is taken as $10(\mathrm{~m}), z$ is the vertical height $(\mathrm{m})$, and the ground roughness index $\alpha$ is 0.15 [36]. The turbulence intensity $I$ and the turbulent integral scale $l(\mathrm{~m})$ are used to determine the inlet profile of the turbulent kinetic energy $k$ and the turbulent diffusion rate $\varepsilon$ : 


$$
\begin{aligned}
& k=1.5(u(z) \cdot I)^{2}, \\
& \mathcal{\varepsilon}=\frac{0.09^{0.75} \cdot k^{1.5}}{l}, \\
& I= \begin{cases}0.1\left(\frac{z_{b}}{z_{G}}\right)^{-\alpha-0.05}, & \left(z \leq z_{b}\right), \\
0.1\left(\frac{z}{z_{G}}\right)^{-\alpha-0.05}, & \left(z_{b} \leq z \leq z_{G}\right), \\
100\left(\frac{z}{30}\right)^{0.5}, & \left(30 \mathrm{~m} \leq z \leq z_{G}\right), \\
100, & (z \leq 30 \mathrm{~m}) .\end{cases}
\end{aligned}
$$

This is set as a II landform according to Japanese regulations [37], where $z_{b}$ is $5(\mathrm{~m})$ and $z_{G}$ is $350(\mathrm{~m})$. The inlet boundary condition for the snow phase on the inflow surface is

$$
f= \begin{cases}\frac{0.68}{\mu_{p} \mu_{*} g} \mu_{* t}\left(\mu_{*}^{2}-\mu_{* t}^{2}\right), & \left(h_{s} \leq z\right), \\ \frac{0.8 \exp \left[-1.55\left(4.78 \mu_{*}^{-0.544}-z^{-0.544}\right)\right]}{\rho_{p}}, & \left(z \leq h_{s}\right),\end{cases}
$$

where $\mu_{p}$ is the snow particle velocity $(\mathrm{m} / \mathrm{s}), \mu_{p}=2.3 u_{* t}$, and the critical height of the transition and suspension layers $h_{s}$ is $0.5(\mathrm{~m})$.

The air and snow phases at the inlet were calculated using UDF interface programming, with fully developed outflow boundary conditions at the outlet. Free-sliding wall conditions were applied to the top, as well as the left and right sides of the watershed, and nonsliding wall conditions were used for the snow surface and the model wall surface. The realisable $k-\varepsilon$ two-equation model was selected for the calculations. In the iterative calculations, a dimensionless root-mean-square residual of $10^{-6}$ is used as the convergence criterion, and a second-order discrete scheme was used for the solution procedure.

3.5. Accuracy Verification. The model from literature [38] is selected for the accuracy verification of the numerical simulation. In the literature, the spherical shell roof has a span $L_{1}$ of $60 \mathrm{~m}$ and the shell height $h$ is $13.2 \mathrm{~m}$, and the distance between the edge of the roof and the ground surface $H$ is $15 \mathrm{~m}$, as shown in Figure 8. The calculated river basin is $720 \mathrm{~m} \times 540 \mathrm{~m} \times 110 \mathrm{~m}$, which is divided using an unstructured grid, with a total of approximately 1.35 million grids. The boundary conditions and other parameters are shown in detail in the literature. The snow distribution pattern of the roof using the same simulation conditions and the literature simulation results are shown in Figure 9.

From Figures 9(a) and 9(b), it can be seen that the roof snow simulated in the paper and the roof snow simulated in literature [38] were both eroded. The erosion amount of the roof snow in the leeward-side edge in the literature was slightly greater than the result obtained in the paper. Both in the literature and in the paper, along the windward-side edge of the reticulated shell to the central part, the absolute value of the snow erosion amount increased gradually from small values, reaching a maximum at the centre, and the patterns of change for the snow erosion amount were consistent. The absolute values of the snow erosion at the centre reported in the literature and obtained in the paper were the same, and both were 0.00055 . This finding proved the accuracy of the numerical simulation used in the paper and provided a basis for the simulation study of the wind-induced snow drift.

\section{Test and Numerical Simulation Results}

4.1. Influence of the Opening Direction. The influence of the direction of the stadium opening (one open end) on the snow distribution on the surface is determined for five working conditions, i.e., the stadium opening directions of $0^{\circ}, 90^{\circ}, 180^{\circ}$, and $270^{\circ}$ and a closed stadium. The locations of the measurement points for the closed stadium are shown in Figure 10, the opening angle and the measurement points for the open stadium are shown in Figure 5(b), and the wind tunnel measurements of the snow distribution on the gymnasium surface are shown in Figure 11. For $C_{s} \geq 1$, snow was deposited on the surface of the structure, and erosion occurred for $C_{s}<1$.

Figure 11 shows that $C_{s}$ first decreased and, then, increased from the windward side to the leeward side of the gymnasium, and a large area of leeward snow was deposited. The peak $C_{s}$ values were found at measurement points 13,33 , 49,61 , and 67, indicating that the greatest snow depth occurred on the central axis of the leeward side. Measurement points $7,19,29,37,45,53,59$, and 63 on the central axis perpendicular to the downwind direction were located in the valley, where the largest amount of snow erosion occurred. Therefore, the variation in the snow pressure at these locations should be considered when designing these structures to prevent structural surface damage from uneven snow pressure.

Compared with the $C_{s}$ value for the closed stadium, larger $C_{s}$ values were found for the $0^{\circ}$ and $180^{\circ}$ openings, and smaller or equal $C_{s}$ values were found for the $90^{\circ}$ opening. Thus, the stadium opening had a significant influence on the snow distribution on the gymnasium surface. Comparing the results for the four opening conditions showed consistent variations in $C_{s}$ for the $0^{\circ}$ and $180^{\circ}$ openings, which were greater than that for the $90^{\circ}$ opening, which was almost entirely below unity. At measurement point 1 on the central axis of the windward edge, $C_{s}$ for the $90^{\circ}$ opening was approximately $38.18 \%$ smaller than those for the $0^{\circ}$ and $180^{\circ}$ openings and approximately $20 \%$ smaller than that for the $270^{\circ}$ opening. At measurement point 25 on the central axis of the windward side, $C_{s}$ for the $90^{\circ}$ opening was less than those for the $0^{\circ}, 180^{\circ}$, and $270^{\circ}$ openings by $35.24 \%, 37.04 \%$, and $18.07 \%$, respectively. At measurement point 13 on the central axis of the leeward edge, $C_{s}$ for the $90^{\circ}$ opening was $18.4 \%$ smaller than that for the $0^{\circ}$ opening and $20.31 \%$ 


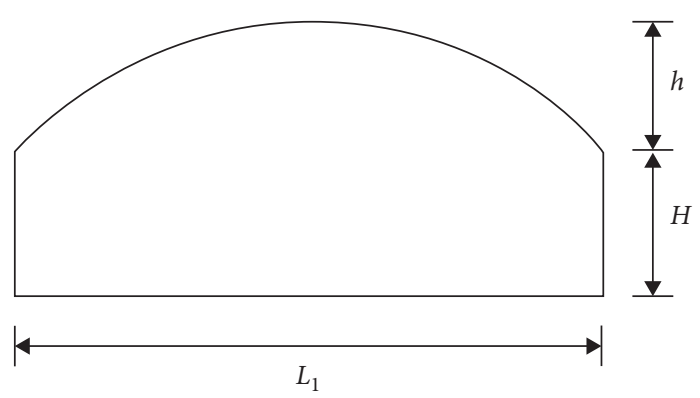

Figure 8: Model of the spherical shell roof.

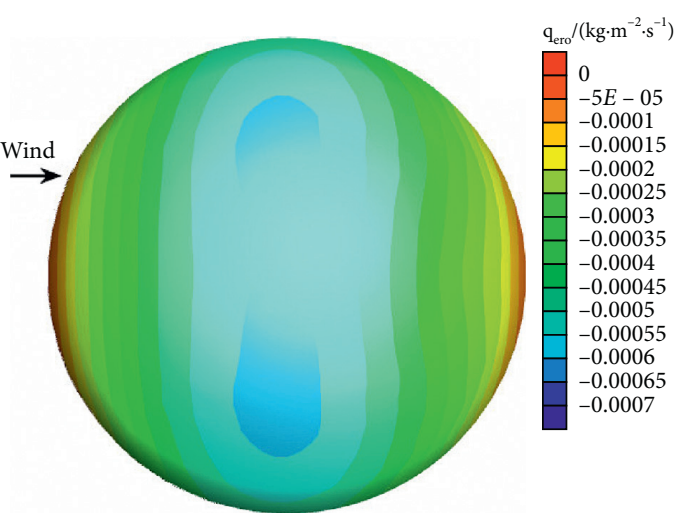

(a)

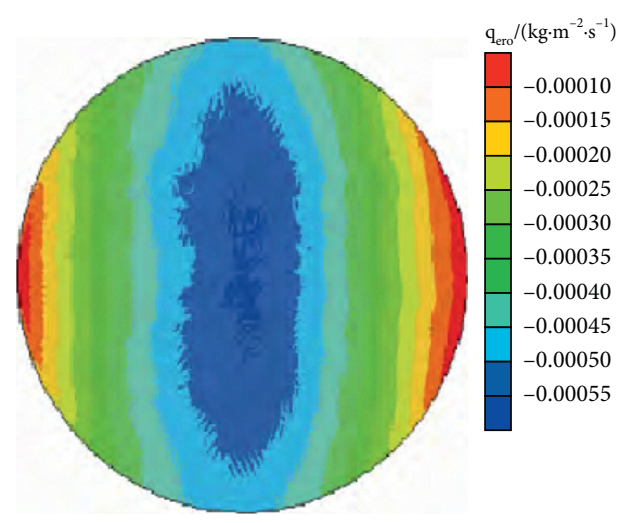

(b)

Figure 9: Comparison of the simulation results. (a) Simulation results of this paper. (b) Simulation results in the literature.

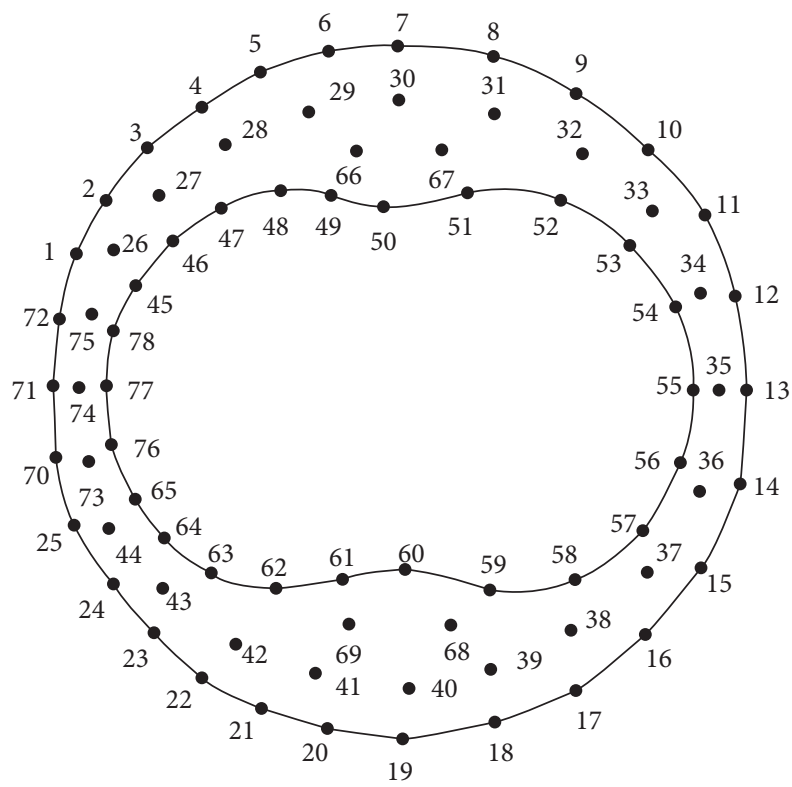

FigURE 10: Layout of the measurement points for a closed stadium.

smaller than those for the $180^{\circ}$ and $270^{\circ}$ openings. At measurement point 33 on the central axis of the leeward side, $C_{s}$ for the $90^{\circ}$ opening was smaller than those for the $0^{\circ}, 180^{\circ}$, and $270^{\circ}$ openings by approximately $20.83 \%, 22.76 \%$, and $21.49 \%$, respectively. The $90^{\circ}$ opening of the stadium directly faced the gymnasium, and there was a relatively large elevation difference between the two buildings. The relatively high wind velocity carried the snow on the windward side of the gymnasium into the air, forming a backflow vortex. Therefore, the variation in the $C_{s}$ values at measurement points 1 to 7 and 19 to 29 on the windward side was different from that for other working conditions. In the leeward area, some snow was also blown off the surface by the relatively high-speed wind, resulting in a smaller $C_{s}$ than for the other working conditions. Therefore, the $90^{\circ}$ stadium opening facilitated the distribution of snow on the gymnasium surface.

The measurement point locations on the stadium surface depended on the working conditions; thus, the four open working conditions were analysed separately from the corresponding closed case. A comparison of the variation in $C_{s}$ is shown in Figure 12.

In Figure 12, all of the peak $C_{s}$ values for the open stadium under different working conditions were obtained on the central axis of the leeward side. The valleys at the boundary of the windward and leeward sides result in the smallest $C_{s}$ on the central axis being perpendicular to the downwind direction. Comparing the results for the open and closed stadiums showed that, for the four working conditions, there was a similar variation in $C_{s}$ over the measurement points on the outermost circle and the second circle, which were considerably different from the $C_{s}$ values for the innermost circle. The wind flowed into the stadium through the opening and 


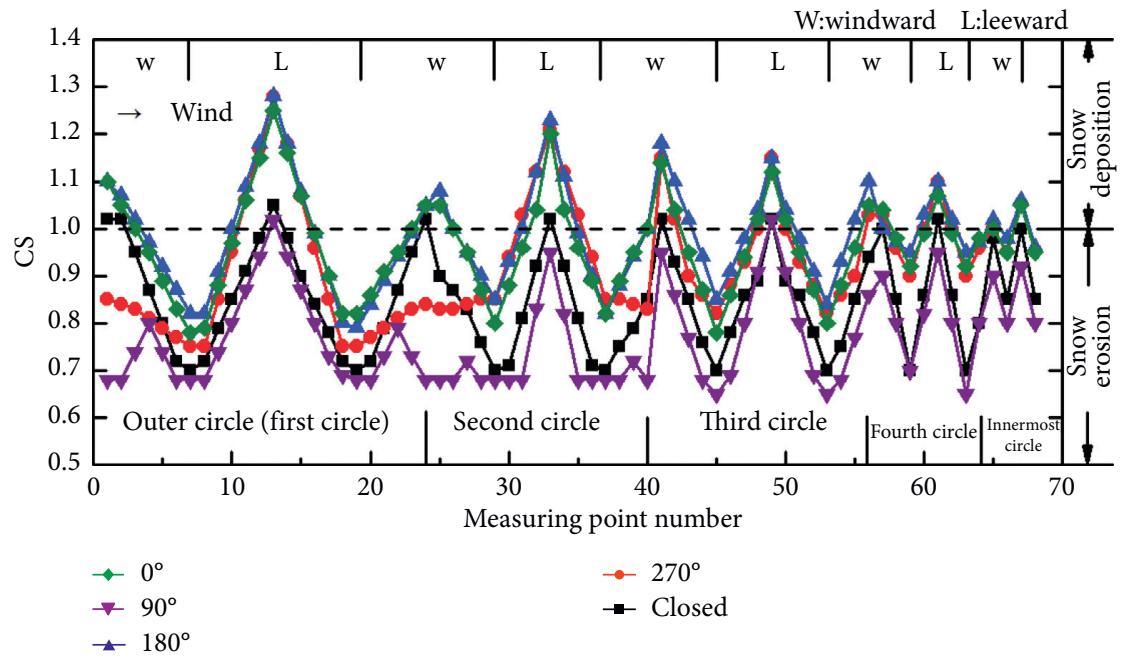

FIgURE 11: Snow distribution on the gymnasium surface for different opening directions.

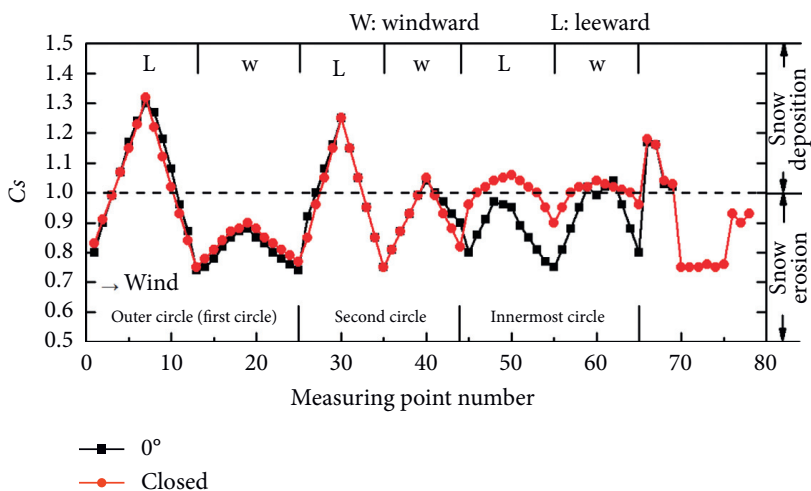

(a)

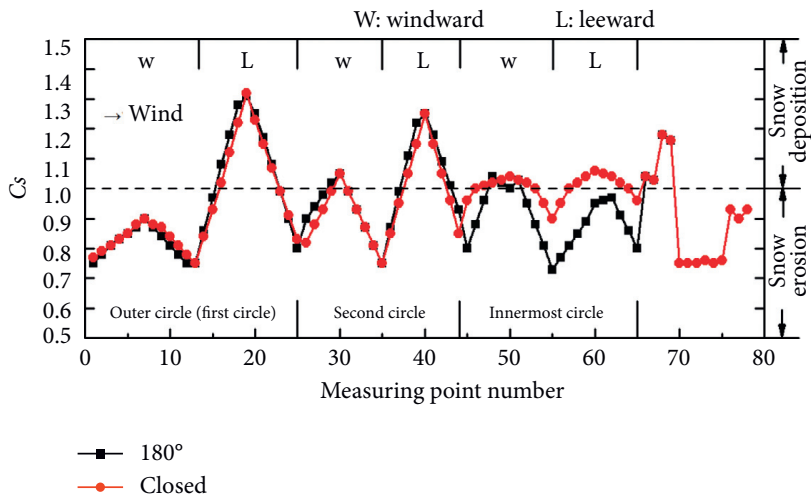

(c)

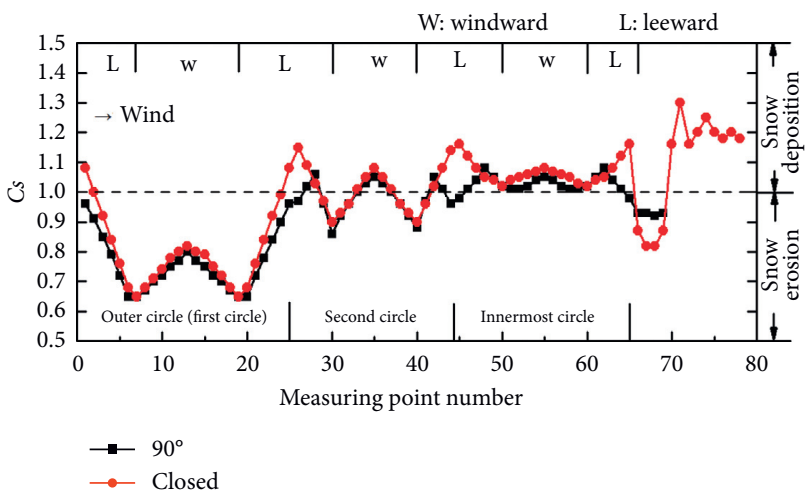

(b)

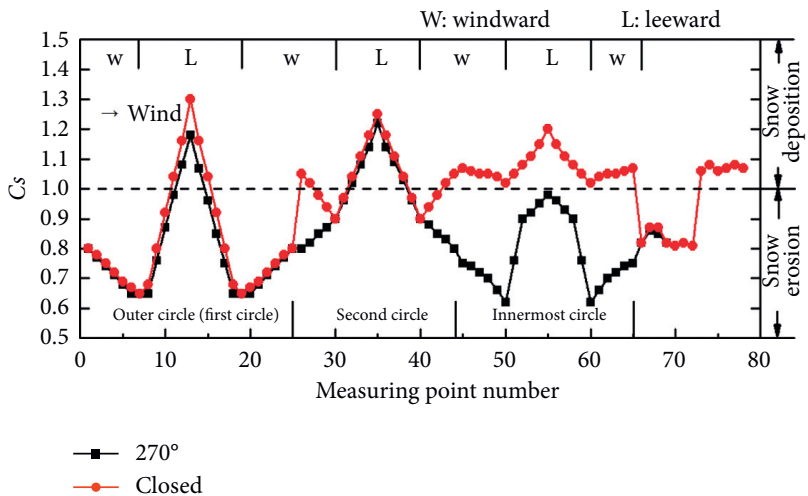

(d)

Figure 12: Comparison of the snow distribution on the surfaces of an open stadium for different opening directions and a closed stadium. (a) $0^{\circ}$. (b) $90^{\circ}$. (c) $180^{\circ}$. (d) $270^{\circ}$.

formed a vortex that carried the snow on the surface away. Therefore, $C_{s}$ for the innermost circle was less than that for the closed stadium. The stadium opening also affected the distribution of snow on the stadium surface.

A comparison of Figure 12(a) and 12(c) showed that, for the $0^{\circ}$ and $180^{\circ}$ openings, the $C_{s}$ values for the outermost circle (measurement points 1 to 25) were symmetric about measurement point 13, the $C_{s}$ values for the second circle (measurement points 26 to 44 ) were symmetric about measurement point 35 , and the $C_{s}$ values for the innermost circle (measurement points 45 to 65 ) were symmetric about measurement point 55 . Thus, the $0^{\circ}$ and $180^{\circ}$ working 
conditions only differed in terms of the opening direction and produced the same surface snow distributions. Less snow was deposited on the surface for the $90^{\circ}$ and $270^{\circ}$ openings than for the $0^{\circ}$ and $180^{\circ}$ openings. For the $270^{\circ}$ opening, there was a considerable difference in $C_{s}$ between the open and closed conditions for the innermost circle (measurement points 45 to 65 ), and the $C_{s}$ at measurement points 50 and 60 for the closed stadium was approximately $39.22 \%$ higher than that for the open stadium. In this case, the opening directly faced the inflow direction, such that most of the wind flowed into the stadium, encountered the inner wall, and flowed back to form a vortex. At this time, the inner wall had no outlet to expel such a high-energy vortex, resulting in small $C_{s}$ values for the innermost circle. In contrast, for the closed stadium, most of the wind flowed along the smooth wall to the leeward side, and the influence of the wind on the inner wall of the stadium was much smaller than for the open stadium. The most uniform snow distribution on the gymnasium surface was obtained for the $90^{\circ}$ opening, and except for the outermost circle, the $C_{s}$ values for the other measurement points varied smoothly and were concentrated between 0.9 and 1.1. The values of $C_{s}<1$ for the measurements points on the outermost circle indicated snow erosion. Thus, the stadium surface would not be damaged by uneven snow distribution.

4.2. Influence of Spacing. Tests were conducted for a gymnasium span of $\mathrm{L}$ and spacing between the stadium and the gymnasium of $0.3 \mathrm{~L}, 0.5 \mathrm{~L}, 1 \mathrm{~L}, 1.5 \mathrm{~L}, 2 \mathrm{~L}$, and $2.5 \mathrm{~L}$. The snow distribution of the two structures was compared to the results of the single models without interference and is shown in Figure 13.

In Figure 13, the same variation in $C_{s}$ was found for the gymnasium and the stadium under the seven working conditions. All $C_{s}$ values decreased with increasing distance $(0.3 \mathrm{~L}-2 \mathrm{~L})$. For the $0.3 \mathrm{~L}$ spacing, the $C_{s}$ values of the two buildings were significantly higher than for $2 \mathrm{~L}$. From Figure 13(a), it can be seen that the measurement points 13 and 33 of the gymnasium surface were on the transversal axis of the leeward side. For measurement point 13, the $C_{s}$ value at $0.3 \mathrm{~L}$ was approximately $19.42 \%$ higher than that at $2 \mathrm{~L}$. For measurement point 33 , the $C_{s}$ value at $0.3 \mathrm{~L}$ was approximately $17.91 \%$ higher than that at $2 \mathrm{~L}$. From Figure 13(b), it follows that the values of $C_{s}$ were significantly higher on the leeward side than those on the windward side for the stadium measurement points for the outer circle and second circle. For measurement point 20, the $C_{s}$ value at $0.3 \mathrm{~L}$ was approximately $16.67 \%$ higher than that at $2 \mathrm{~L}$. For measurement point 40 , the $C_{s}$ value at $0.3 \mathrm{~L}$ was approximately $19.85 \%$ higher than that at $2 \mathrm{~L}$. Thus, under different spacings, the wind-induced snow drift had a mutual influence on the adjacent long-span buildings; that is, both the blocked (gymnasium) and blocking (stadium) structures were affected. For spacings of $2 \mathrm{~L}$ and $2.5 \mathrm{~L}$, the $C_{s}$ values of both surfaces were essentially the same as without interference.

The reason for this is that there was a large difference in the heights between the stadium and gymnasium, and the wind formed a reflux vortex between the two structures that entrapped the ground particles in the air before they landed on the roof. This resulted in a larger $C_{s}$ value for the roof. As the structure spacing increased, the energy of the vortex decreased and caused the $C_{s}$ value of the roof to decrease accordingly. When the distance was large enough, the wind flow changed to blow around the gymnasium. Therefore, for a sufficiently large distance between two long-span structures, the mutual interference effect of the wind-induced snow motion between the structures was negligible.

4.3. Influence of the Wind Direction Angle. Wind tunnel tests were used to study the stadium's influence on the snow distribution on the gymnasium surface for different wind direction angles. For several wind direction angles varying from $0^{\circ}$ to $360^{\circ}$ with an interval of $45^{\circ}$. The wind direction angle is shown in Figure 6(b). The snow distribution pattern on the gymnasium surface for the eight working conditions is shown in Figure 14.

It can be seen from Figure 14 that compared to the two influencing factors, that is, the opening direction and structure spacing, the wind direction angle had the greatest influence on the snow distribution on the surface of the gymnasium. The snow on the surface of the gymnasium under a wind direction angle of $270^{\circ}$ had the largest erosion area, which was the most structurally safe and beneficial. The erosion of snow under the $225^{\circ}$ wind direction angle and $315^{\circ}$ wind direction angle was also relatively serious. The reason is that, for these three wind angles, the gymnasium was located upstream of the stadium. The incoming wind blew directly on the surface of the gymnasium, and the stadium did not play a blocking role. The snow deposition areas at the $225^{\circ}$ and $315^{\circ}$ wind direction angles were not as large as the erosion area, but the snow thickness at the deposition site was also extremely large. This results in a significant uneven distribution of snow on the surface of the gymnasium, which was detrimental to the structure. The trends in $C_{s}$ for wind direction angles of $0^{\circ}$ and $180^{\circ}$ were approximately the same. Under the $45^{\circ}$ and $135^{\circ}$ wind direction angles, a large area of snow deposit occurred, and the $C_{s}$ value was between 1.1 and 1.3. Excessive snow can easily cause the roof to collapse. It is believed that the stadium was at the upstream of the gymnasium at this time. Due to the blocking effect of the stadium, the incoming wind produced a complex turbulence, which was extremely detrimental to the safety of the gymnasium.

In the study of the distribution regularity of wind-induced snow motion, the wind direction angles were a very important factor. However, in the wind tunnel test, it was impossible to quantitatively study the influence of the stadium with one open end on the snow distribution on the gymnasium surface and the mutual interference effect between them. Therefore, a numerical simulation was adopted to supplement this influence factor. This method can more intuitively reflect the influence of the wind direction angle on the surface snow of the gymnasium, with an interference coefficient, Ic: 


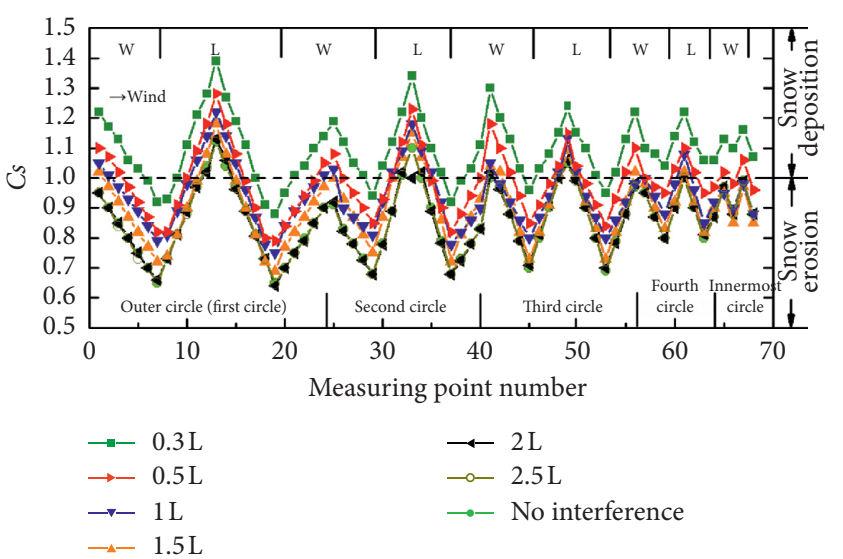

(a)

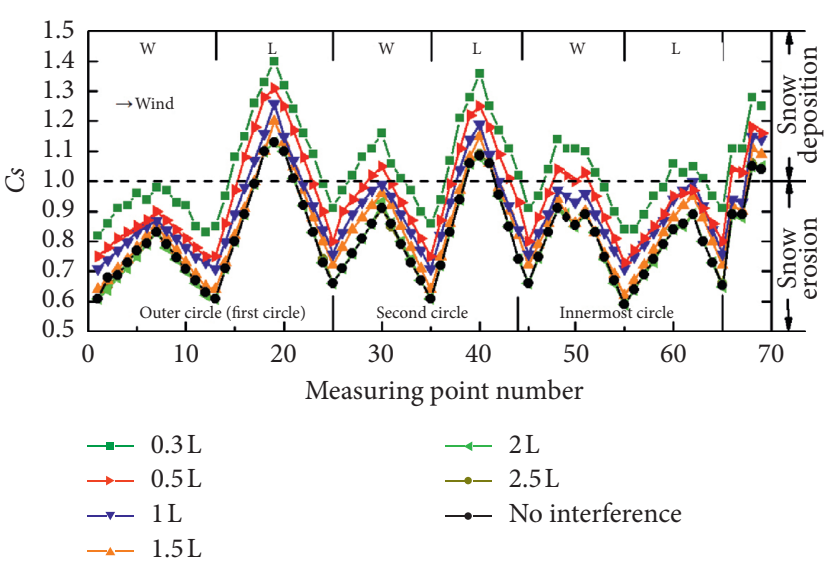

(b)

Figure 13: Snow distribution on the gymnasium and stadium surfaces for different spacings between structures. (a) Gymnasium. (b) Stadium.

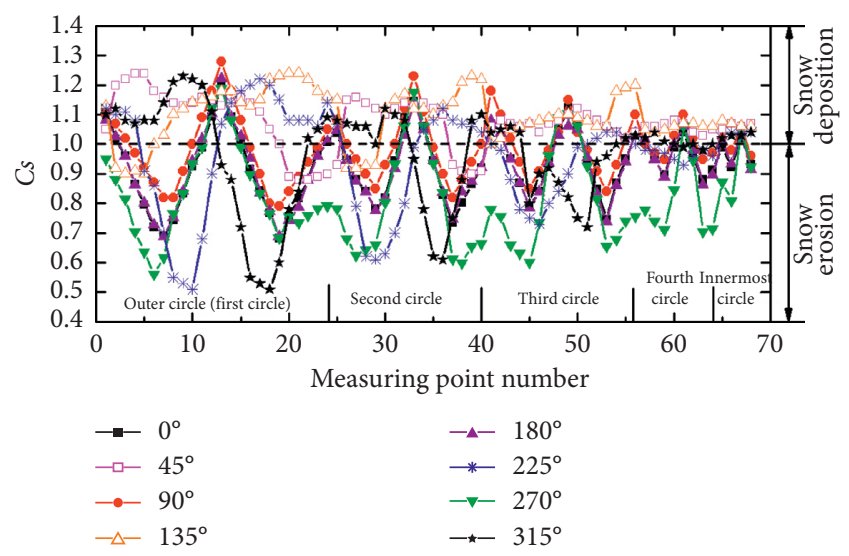

FIGURE 14: Snow distribution on the gymnasium surface under different wind direction angles.

$$
I c=\frac{q^{\prime}}{q},
$$

where $q^{\prime}$ is the snow cover on the gymnasium surface and $q$ is the cover of unaffected snow. To facilitate the analysis, we define $I c=1$ as a line of demarcation, where $I c \leq 1$ corresponds to blockage interference and $I c>1$ indicates an amplification interference. A large value of $|I c-1|$ implies a significant interference effect on the gymnasium surface.

The simulation accuracy is verified by comparing the results of a numerical simulation for a $180^{\circ}$ gymnasium opening with the test results, as shown in Figure 15.

In Figure 15, the numerical simulation results were larger than the test results, which is explained as follows. First, it was difficult to accurately simulate the wind tunnel test environment by using only the wind profile and roughness at the inlet in the numerical simulation. The boundary conditions also impacted the results by creating vortexes and separation in the airflow around the model. Second, a scale model was used in the wind tunnel test, whereas a full-scale model was numerically simulated. Third, the realisable $k-\varepsilon$ turbulence model used in the numerical simulation may not be able to accurately calculate the wind pressure in regions of high negative pressure. The aforementioned considerations produced the deviations between the results of the wind tunnel test and the numerical simulation. However, the variation in $C_{s}$ was essentially the same for the test and simulation results, indicating that the numerical simulation was reasonably accurate.

To create a clearer analysis of the interference coefficient $I c$, the $I c$ of the gymnasium under the eight groups of wind direction angles are divided into groups for research. These groups are the working conditions of the gymnasium downstream of the downwind $\left(45^{\circ}, 90^{\circ}\right.$, and $\left.135^{\circ}\right)$, the gymnasium upstream of the downwind $\left(225^{\circ}, 270^{\circ}\right.$, and $315^{\circ}$ ), and the wind direction perpendicular to the transverse axis of the gymnasium $\left(0^{\circ}\right.$ and $\left.180^{\circ}\right)$. The wind direction is defined in Figure 6(b), and the obtained interference coefficients are compared in Figure 16.

From Figure 16(a), it can be seen that when the wind direction angles were $45^{\circ}, 90^{\circ}$, and $135^{\circ}$, Ic $>1$, indicating that under this group of wind direction angles, the impact of the stadium on the gymnasium was mainly amplification interference. For the $45^{\circ}$ and $135^{\circ}$ wind direction angles, the peaks of $I c$ were on the longitudinal axis of the gymnasium. Attention should be given to the impact of snow on the roof. These points had Ic values far greater than the $90^{\circ}$ wind direction angle. For measurement point 7, the value of Ic at $90^{\circ}$ was smaller than that at $45^{\circ}$ and $135^{\circ}$ by $19.23 \%$ and $17.10 \%$, respectively. For measurement point 19, these values were $18.24 \%$ and $16.55 \%$ smaller. For this group of wind direction angles, the $45^{\circ}$ and $135^{\circ}$ wind direction angles had more significant interference effects than the $90^{\circ}$ wind direction angle, which were more unfavourable for structural safety.

Figure 16(b) shows that there was both amplification interference and blockage interference under the wind direction angles of $225^{\circ}, 270^{\circ}$, and $315^{\circ}$. Amplification interference was in the area close to the stadium, and blockage interference mainly occurred in the windward area of the 


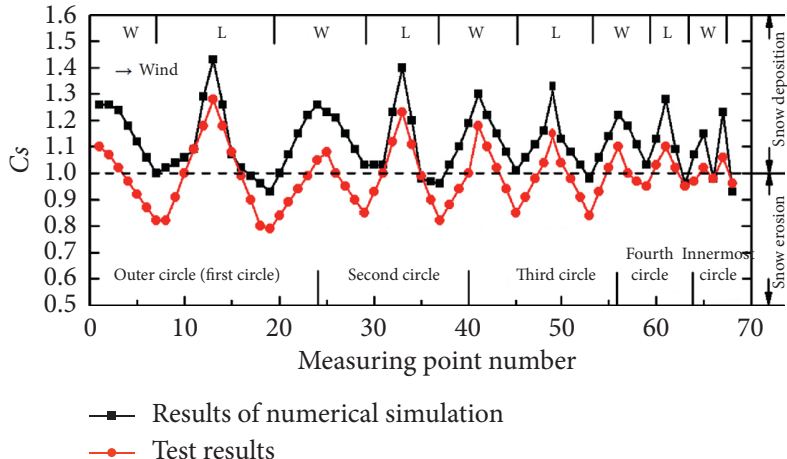

(a)

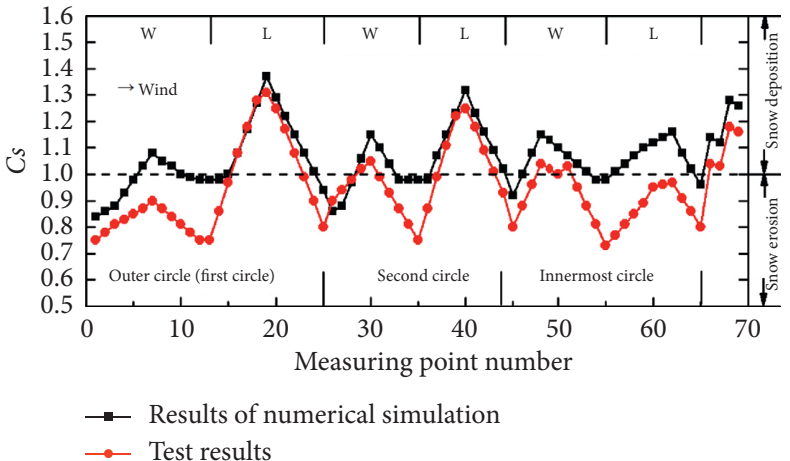

(b)

FIgURE 15: Comparison of the results from tests and simulations. (a) Gymnasium. (b) Stadium.

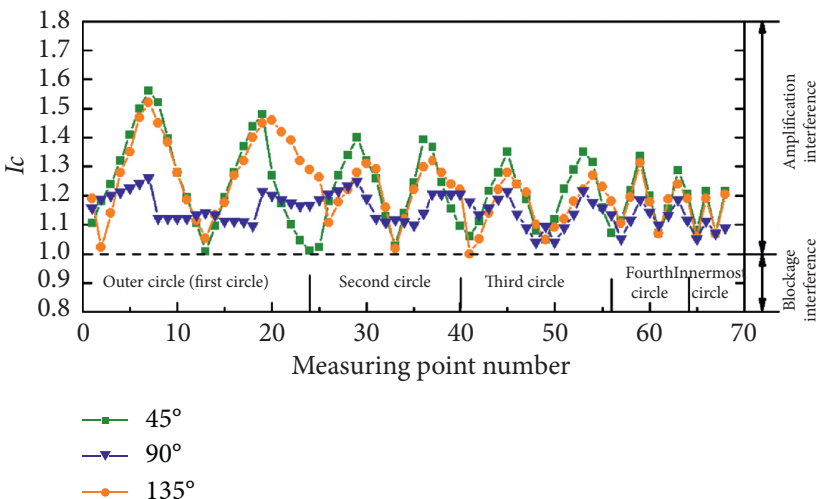

(a)

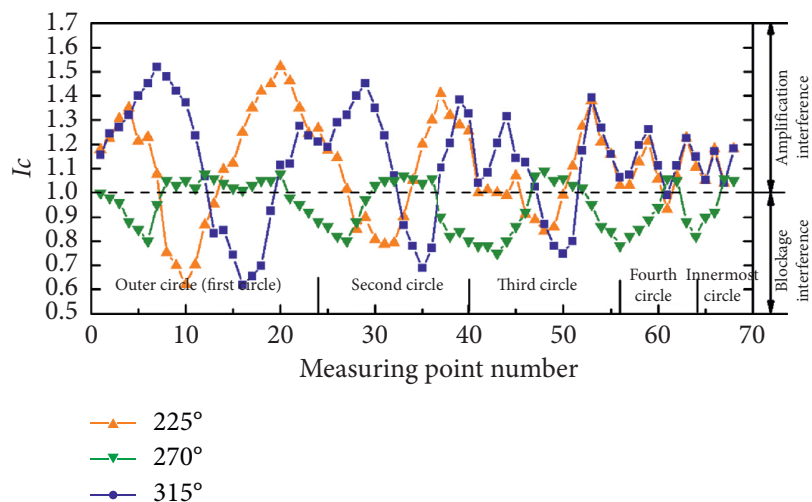

(b)

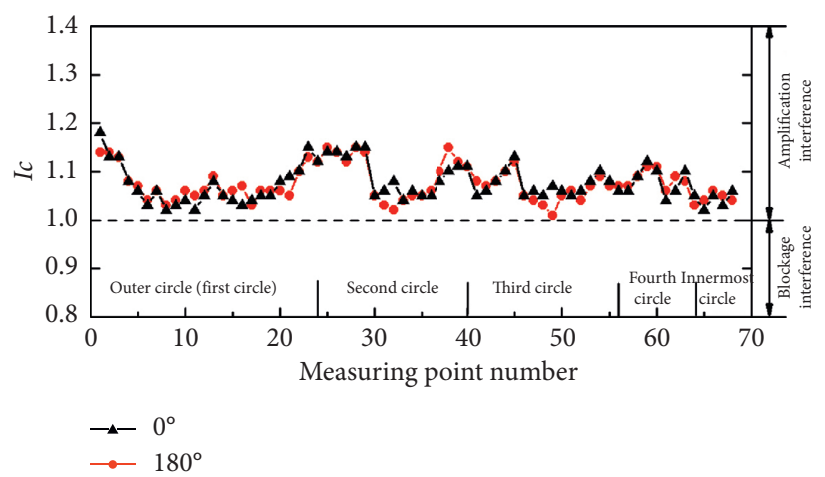

(c)

FiguRE 16: Interference coefficients for the gymnasium surface for different wind direction angles. (a) $45^{\circ}, 90^{\circ}$, and $135^{\circ}$. (b) $225^{\circ}, 270^{\circ}$, and $315^{\circ}$. (c) $0^{\circ}$ and $180^{\circ}$.

roof. At the $225^{\circ}$ and $315^{\circ}$ wind direction angles, the maximum value of $I c$ was 1.52 , and the minimum value of $I c$ was 0.62 . Due to the enormous difference between the $I c$ values under the two working conditions, the snow distribution on the roof was extremely uneven and could easily cause roof damage. The amplification interference effect at the $270^{\circ}$ wind direction angle was very small. The maximum value of Ic was 1.08 , and the blocking effect was obvious. The minimum value of $I c$ was 0.75 , which was beneficial to structural safety.

From Figure 16(c), it can be seen that the trends of changes for $I c$ under the wind direction angles of $0^{\circ}$ and $180^{\circ}$ were roughly the same, and $I c>1$, indicating that the impact of the stadium on the gymnasium was mainly amplification interference. However, the maximum value of $I c$ at the $0^{\circ}$ wind direction angle was 1.18 , and the maximum value of $I c$ 
at the $180^{\circ}$ wind direction angle was 1.15 . In summary, the amplification interference effect of the stadium on the gymnasium under the wind direction angles of $0^{\circ}$ and $180^{\circ}$ was far less than that under the wind direction angles of $45^{\circ}$, $90^{\circ}$, and $135^{\circ}$. Based on the abovementioned figures, it can be concluded that the amplification interference effect under the $45^{\circ}$ wind direction angle was particularly serious, which was extremely unfavourable to the roof's stability, while the $270^{\circ}$ wind direction angle was the most beneficial to structural safety. This conclusion was consistent with the results of the wind tunnel tests under different wind direction angles.

\section{Discussion}

Wind tunnel tests and numerical simulations are used to study snow distribution on stadium and gymnasium roofs. Although the results of the two studies are numerically different, the same snow variation trends are obtained. Thus, the simulations can reasonably reflect the snow distribution on the roofs of the two structures. Based on the conclusions obtained from the experiments and simulations, this paper discussed the aspects of different opening angles and wind direction angles of open stadiums and gymnasiums, as well as how to reasonably avoid snow damage in large-span buildings.

The open end in the stadium (i.e., the blocking structure) had a considerable impact on the snow distribution on the roof of the gymnasium (i.e., the blocked structure). The snow drift on the gymnasium surface also impacted the snow distribution on the stadium surface. That is, there were mutual interference effects due to the motion of snow between the two structures. The stadium was a smooth, longspan structure, which was highly sensitive to wind. An opening at one end of the structure created a pressure difference between the interior and exterior spaces divided by the opening. This pressure gradient drove the wind flow into and around the structure. The wind flow field varied with the direction of the opening, and the snow distribution on the roofs of the two structures varied accordingly. The snow distribution on the ground inside the stadium also changed. In future design studies, excessive snow in the stadium should be prevented to not to affect normal stadium use.

For wind direction angles of $45^{\circ}$ and $270^{\circ}$, there was an enormous difference in the $I c$ values of the two structures. For a wind angle of $45^{\circ}$, the stadium was upstream of the gymnasium and blocked the gymnasium. The relatively large height difference between the two structures caused the wind to flow directly from the leeward side of the stadium into the centre of the gymnasium, resulting in an Ic value of between 1 and 1.56 for a wind direction angle of $90^{\circ}$. For a wind direction angle of $270^{\circ}$, the gymnasium was upstream of the stadium. Since the stadium was much higher than the gymnasium, the wind flowing from the leeward side of the gymnasium was blocked by the relatively high stadium and formed a backflow vortex that carried the snow on the leeward side of the gymnasium away, resulting in $I c<1$.
For functional buildings such as stadiums and gymnasiums where people are concentrated, avoiding snow disasters has always been a focus of research. There are many factors that affect snow distribution on the surface of such large-span structures, among which are the opening direction of the stadium, structure spacing, wind direction angle, temperature, and viscosity of the snow particle. The focus of the present paper is on the first three factors. Concerning snow distribution, the wind direction angle has the greatest effect and spacing has the least effect. For such large-span building complexes, our study indicates that the designer should try to ensure that the lower buildings are located upstream of the incoming wind. If there are openings in the building, they should be oriented towards the other large-span building, and the two structures should be separated far enough.

\section{Conclusions}

Wind tunnel tests were conducted to study the influence of the direction of a stadium opening and the spacing, as well as the wind angle between the stadium and gymnasium on the snow distribution on the structures' surfaces. Numerical simulations were conducted to analyse the corresponding influence of the wind direction angle. The following conclusions are drawn:

(1) A study of the snow distribution on the roof of a long-span structure should include the influence of the surrounding structures. For buildings that are open at one end, a vortex can easily form in the interior. Thus, the internal wind-induced snow flow and snow distribution should be considered.

(2) The stadium opening significantly impacted the snow distribution on the gymnasium surface. A stadium opening that directly faced the gymnasium was the most favourable orientation for distributing snow over the surfaces of the two structures.

(3) The mutual interference effect between the stadium and gymnasium decreased as the spacing between the two structures increased. This effect could be neglected when the spacing between the two structures was twice the gymnasium span.

(4) The interference effect of the stadium on the gymnasium depended on the wind direction angle. The amplification interference effect was most significant for a wind direction angle of $45^{\circ}$. Both the amplification and blockage interference come into play for a wind direction angle of $270^{\circ}$.

(5) Considering the effect of various factors on the mutual interference effect of the wind-induced snow motion of adjacent long-span open stadiums provided a reasonable basis for future structural designs of open buildings under wind-induced snow action.

\section{Data Availability}

The data used to support the findings of this study are available from the first author upon request. 


\section{Conflicts of Interest}

The authors declare that they have no conflicts of interest.

\section{Acknowledgments}

This research was supported by the National Natural Science Foundation of China (grant no. U1261122).

\section{References}

[1] R. A. Schmidt, "Threshold wind-speeds and elastic impact in snow transport," Journal of Glaciology, vol. 26, no. 94, pp. $453-467,1980$.

[2] R. J. Kind, "Concentration and mass flux of particles in aeolian suspension near tailings disposal sites or similar sources," Journal of Wind Engineering and Industrial Aerodynamics, vol. 41, no. 1-3, pp. 217-225, 1992.

[3] J. Cocca and M. O'Rourke, "Mathematical simulation of 50year snow drift loads," in Proceedings of the 2008 Structures Congress-Structures Congress 2008: Crossing the Borders, pp. 1-9, ASCE, Vancouver, CA, USA, April 2008.

[4] M. J. O’Rourke, R. S. Speck, and U. Stiefel, "Drift snow loads on multilevel roofs," Journal of Structural Engineering, vol. 111, no. 2, pp. 290-306, 1985.

[5] S. Oikawa, T. Tomabechi, and T. Ishihara, "One-day observations of snowdrifts around a model cube," Journal of Snow Engineering of Japan, vol. 15, no. 4, pp. 283-291, 1999.

[6] T. K. Thiis, "Large scale studies of development of snowdrifts around buildings," Journal of Wind Engineering and Industrial Aerodynamics, vol. 91, no. 6, pp. 829-839, 2003.

[7] T. Tomabechi, K. Hosokawa, and M. Tsuchiya, "Field measurement of snowdrift on a two-level flat roof model," Journal of the Japanese Society of Snow and Ice, vol. 65, no. 3, pp. 231-239, 2003.

[8] R. J. Kind, "A critical examination of the requirements for model simulation of wind-induced erosion/deposition phenomena such as snow drifting," Atmospheric Environment, vol. 10, no. 3, pp. 219-227, 1976.

[9] R. D. Tabler, "Self-similarity of wind profiles in blowing snow allows outdoor modeling," Journal of Glaciology, vol. 26, no. 94, pp. 421-434, 1980.

[10] J. D. Iversen, "Comparison of wind-tunnel model and fullscale snow fence drifts," Journal of Wind Engineering and Industrial Aerodynamics, vol. 8, no. 3, pp. 231-249, 1981.

[11] Y. Anno, "Applications of Anno's modeling conditions to outdoor modeling of snowdrifts," Cold Regions Science and Technology, vol. 9, no. 2, pp. 179-181, 1984.

[12] D. H. Kim, K. C. S. Kwok, D. J. Smedley, and H. F. Rohde, "Modelling of snowdrift around prismatic buildings for antartic environment," International Journal of Offshore and Polar Engineering, vol. 2, no. 1, pp. 73-79, 1992.

[13] K. C. S. Kwok, D. H. Kim, D. J. Smedley, and H. F. Rohde, "Snowdrift around buildings for antarctic environment," Journal of Wind Engineering and Industrial Aerodynamics, vol. 44, no. 1-3, pp. 2797-2808, 1992.

[14] J. H. Lever and R. Haehnel, "Scaling snowdrift development rate," Hydrological Processes, vol. 9, no. 8, pp. 935-946, 1995.

[15] J. A. Peterka and W. S. Esterday, "Roof design snow loads by wind tunnel test and analysis," in Proceedings of the Structures 2004 at Building on the Past, Securing the Future, pp. 1-9, ASCE, Nashville, TN, USA, May 2004.

[16] X. Zhou, L. Kang, M. Gu, L. Qiu, and J. Hu, "Numerical simulation and wind tunnel test for redistribution of snow on a flat roof," Journal of Wind Engineering and Industrial Aerodynamics, vol. 153, pp. 92-105, 2016.

[17] X. Zhou, L. Kang, X. Yuan, and M. Gu, "Wind tunnel test of snow redistribution on flat roofs," Cold Regions Science and Technology, vol. 127, pp. 49-56, 2016.

[18] Z. X. Yu, F. Zhu, R. Cao et al., "Wind tunnel tests and CFD simulations for snow redistribution on roofs 3D stepped flat roofs," Wind and Structures An International Journal, vol. 28, pp. 31-47, 2019.

[19] S. Qiang, X. Zhou, K. Kosugi, and M. Gu, "A study of snow drifting on a flat roof during snowfall based on simulations in a cryogenic wind tunnel," Journal of Wind Engineering and Industrial Aerodynamics, vol. 188, pp. 269-279, 2019.

[20] T. Sato, T. Uematsu, T. Nakata, and Y. Kaneda, "Three dimensional numerical simulation of snowdrift," Computational Wind Engineering 1, vol. 46-47, pp. 741-746, 1993.

[21] M. Beyers and B. Waechter, "Modeling transient snowdrift development around complex three-dimensional structures," Journal of Wind Engineering and Industrial Aerodynamics, vol. 96, no. 10-11, pp. 1603-1615, 2008.

[22] L. Zhao, Z. Yu, F. Zhu, X. Qi, and S. Zhao, "CFD-DEM modeling of snowdrifts on stepped flat roofs," Wind and Structures, vol. 23, no. 6, pp. 523-542, 2016.

[23] Z. Liu, Z. Yu, F. Zhu, X. Chen, and Y. Zhou, "An investigation of snow drifting on flat roofs: wind tunnel tests and numerical simulations," Cold Regions Science and Technology, vol. 162, pp. 74-87, 2019.

[24] J. Wang, H. Liu, Z. Chen, and K. Ma, "Probability-based modeling and wind tunnel test of snow distribution on a stepped flat roof," Cold Regions Science and Technology, vol. 163, pp. 98-107, 2019.

[25] C. Marsh, J. Pomeroy, R. Spiteri, and H. Wheater, "A finite volume blowing snow model for use with variable resolution meshes," Water Resources Research, vol. 56, Article ID e2019WR025307, 2020.

[26] B. He, L. Ding, and D. Prasad, "Enhancing urban ventilation performance through the development of precinct ventilation zones: a case study based on the greater Sydney, Australia," Sustainable Cities and Society, vol. 47, pp. 1-14, 2019.

[27] X. Jiang, Z. Yin, and H. Cui, "Wind tunnel tests of windinduced snow distribution for cubes with holes," Advances in Civil Engineering, vol. 2019, no. 1, 12 pages, Article ID 4153481, 2019.

[28] R. J. Kind, "Snowdrifting: a review of modelling methods," Cold Regions Science and Technology, vol. 12, no. 3, pp. 217228, 1986.

[29] X. Li, Research on Snowdrifting on Building Roof and Around Building, Tongji University, Shanghai, China, 2011.

[30] W. Wang, H. Liao, and M. Li, "Wind tunnel test on windinduced roof snow distribution," Journal of Building Structures, vol. 35, no. 5, pp. 135-141, 2014.

[31] X. Zhou, J. Hu, and M. Gu, "Wind tunnel test of snow loads on a stepped flat roof using different granular materials," Natural Hazards, vol. 74, no. 3, pp. 1629-1648, 2014.

[32] X. Li, J. Zhu, Y. Jia et al., "Formation process of extraordinarily serious snowstorm and its induced damage in 2007 in Liaoning Province," Chinese Journal of Ecology, vol. 26, pp. 1250-1258, 2007.

[33] T. Uematsu, T. Nakata, K. Takeuchi, Y. Arisawa, and Y. Kaneda, "Three-dimensional numerical simulation of snowdrift," Cold Regions Science and Technology, vol. 20, no. 1, pp. 65-73, 1991.

[34] Y. Tominaga, T. Okaze, and A. Mochida, "CFD modeling of snowdrift around a building: an overview of models and 
evaluation of a new approach," Building and Environment, vol. 46, no. 4, pp. 899-910, 2011.

[35] P.-A. Sundsbø, "Numerical simulations of wind deflection fins to control snow accumulation in building steps," Journal of Wind Engineering and Industrial Aerodynamics, vol. 74-76, pp. 543-552, 1998.

[36] China National Standards, Load Code for the Design of Building Structures, GB 50009-2012, 2012.

[37] AIJ, Recommendations for Loads on Buildings, Architectural Institute of Japan, Tokyo, Japan, 2004.

[38] Y. Li and X. Yuan, "Numerical simulation of snow drifting and research on snow uneven distribution coefficient of longspan spherical shell roofs," Journal of Building Structures, vol. 35, no. 10, pp. 130-136, 2014. 